\section{Fortalecimento muscular em adolescentes com paralisia cerebral: avaliação de dois protocolos em desenho experimental de caso único}

\section{Muscle strengthening in adolescents with cerebral palsy: an evaluation of two single- case experimental design protocols}

\begin{abstract}
Objectives: to describe changes in the motor function of two adolescents with spastic diplegia, one of them participating in a muscle strengthening in isolation (MSI) protocol, the other in a functional tasks strengthening (FTS) protocol.

Methods: a single case experimental design is a method that reveals the pattern of changes over time during and after therapy. The design included baseline, intervention and four- six- and three-week follow-up phases. The participants were two adolescents with spastic diplegia, each undergoing an intervention protocol: muscle strengthening in isolation (MSI) or functional tasks strengthening (FTS). The intervention protocols included isolated muscle strengthening of the hip and knee extensors and plantar flexors (for the MSI participant), or functional exercises for the same musculatures (for the FTS participant). Weekly measurements were taken of muscle strength, walking speed, time taken to walk up and down stairs, time taken to get up off the floor and time on the Timed Get Up and Go test. The data were analyzed using Celeration Line, Two Standard Deviation Amplitude and Visual Analysis.

Results: gains in muscle strength in the lower limbs were observed in both adolescents. Changes in motor activity were observed in two of the four tasks tested in only one of the adolescents $(p<0.05)$.

Conclusions: The functional specificity of the FTS protocol produced modest effects on performance of motor activities in one of the adolescents with CP, in addition to the gains in muscle strength produced by both protocols.
\end{abstract}

Key words Rehabilitation, Resistance training, Cerebral palsy, Motor activity
Sheyla Rossana Cavalcanti Furtado 1

Daniela Virgínia Vaz 2

Leandro Barbosa de Moura 3

Tatiana Pessoa da Silva Pinto 4

Marisa Cotta Mancini 5

1,2-4 Departamento de Fisioterapia. Escola de Educação Física, Fisioterapia e Terapia Ocupacional. Universidade Federal de Minas Gerais. Belo Horizonte, Minas Gerais, Brasil

5 Programa de Pós-Graduação em Ciências da Reabilitação. Escola de Educação Física, Fisioterapia e Terapia Ocupacional. Universidade Federal de Minas Gerais. Av. Pres. Antônio Carlos, 6627 Campus Pampulha. Belo Horizonte, MG, Brasil. CEP: 31.270-901.

E-mail: mcmancini@ufmg.br

\section{Resumo}

Objetivos: descrever mudanças na função motora de dois adolescentes com diplegia espástica, sendo um deles participante de um protocolo de fortalecimento muscular isolado (FMI) e o outro de protocolo de fortalecimento com tarefas funcionais (FTF).

Métodos: desenho experimental de caso único, metodologia que revela a temporalidade do perfil de mudanças ao longo e após a terapia. Este desenho incluiu fases de baseline, de intervenção e de followup com quatro, seis e três semanas, respectivamente. Participantes foram duas adolescentes com displegia espástica submetidas, cada uma, a um protocolo de intervenção: fortalecimento muscular isolado (FMI) ou fortalecimento com tarefas funcionais (FTF). Os protocolos de intervenção incluíram exercícios de fortalecimento muscular isolado de extensores de quadril, extensores de joelho e flexores plantares (para a participante do FMI), ou de exercicios funcionais para as mesmas musculaturas (para a participante do FTF). Mensurações três vezes por semana avaliaram força muscular, velocidade de marcha, tempo para subir e descer escadas, tempo para se levantar do chão e tempo no teste Timed Get Up and Go. Os dados foram analisados com Celeration Line, Amplitude de Dois Desvios Padrão e Análise Visual.

Resultados: ganhos de força muscular de membros inferiores foram observados nas adolescentes submetidas a ambos os protocolos. Mudanças nas atividades motoras foram observadas em 2 das 4 tarefas testadas em somente uma das adolescentes $(p<0,05)$

Conclusões: a especificidade funcional do protocolo de fortalecimento FTF produziu modestos efeitos no desempenho de atividades motoras de uma das adolescentes com PC, em acréscimo aos ganhos de força muscular produzidos por ambos os protocolos. Palavras-chave Reabilitação, Treinamento de resistência, Paralisia cerebral, Atividade motora 


\section{Introdução}

A paralisia cerebral (PC) é definida como um conjunto de desordens permanentes da postura e do movimento corporal decorrentes de lesões não progressivas em regiões do cérebro durante os períodos pré, peri, ou pós-natal. Tais comprometimentos são usualmente acompanhados de déficits sensoriais, perceptivos, cognitivos e comportamentais. ${ }^{1}$

A forma mais prevalente de PC é a espástica, ${ }^{2}$ sendo que a diplegia espástica acomete aproximadamente $40 \%$ das crianças. ${ }^{3}$ Nos portadores da diplegia espástica, a fraqueza dos músculos flexores plantares, extensores de quadril e do joelho é expressiva. ${ }^{3}$ Crianças com diagnóstico de diplegia espástica podem adquirir a marcha, entretanto, essas crianças apresentam um padrão marcadamente distinto do padrão de marcha normal, como adução e rotação interna do quadril, acrescidos de flexão dos joelhos e flexão plantar. Tal padrão fequentemente resulta em menor velocidade e maior gasto energético na deambulação, 4 impondo ainda dificuldades no desempenho de atividades motoras grossas mais complexas, 5 como descer e subir escadas, saltar e correr. ${ }^{6}$ Com o desenvolvimento dessa criança, especialmente durante a adolescência, as dificuldades funcionais se exacerbam, uma vez que o crescimento corporal ocorre de maneira desproporcional aos ganhos de força muscular.7 Apesar da PC se caracterizar por uma lesão não-progressiva, muitas vezes, os acometimentos motores se intensificam com o tempo. 1,2

A capacidade do indivíduo de executar tarefas ou ações em um determinado ambiente indica o seu nível funcional ou o grau de incapacidade. Segundo a Classificação Internacional de Funcionalidade, Incapacidade e Saúde (CIF), ${ }^{8}$ o grau de funcionalidade ou incapacidade do indívíduo resulta da interação entre sua condição de saúde e os componentes de estrutura e função do corpo, atividade e participação, sobre a influência direta dos fatores ambientais e pessoais. As estruturas e funções corporais (i.e., intrínsecas) se referem às partes anatômicas do corpo e suas funções fisiológicas. Atividade é a execução de uma tarefa ou ação por um indivíduo, enquanto participação é o envolvimento do indivíduo em uma situacão da vida real. Considerando a CIF, a fraqueza muscular tem sido identificada como um componente da estrutura e função corporal frequentemente associada a limitações de atividades funcionais em crianças com PC, sendo necessária a adoção de diferentes estratégias motoras para compensar tal dificuldade.2,5,6,9-11
Por muitos anos, intervenções voltadas ao fortalecimento muscular foram contra indicadas para crianças e adolescentes com paralisia cerebral do tipo espástica, devido à preocupação com o efeito negativo do esforço sobre a espasticidade e padrões de movimento. ${ }^{12}$ Vários fatores têm contribuído para uma mudança de conduta em relação à indicação de exercício físico resistido em crianças com PC.13 Estudos que avaliaram o efeito de exercícios de fortalecimento muscular isolado em crianças com PC não relataram resultados adversos sobre os padrões de movimento, 10 flexibilidade ou espasticidade. 14 Tais evidências influenciaram a prática corrente do treinamento muscular nesses indivíduos. ${ }^{13}$ Diversos protocolos de fortalecimento muscular têm mostrado ganhos significativos na força muscular, $5,6,9,11,15$ redução do gasto energético,4,5,7,11 aumento na velocidade de marcha4,6,9,11,15 e redução no tempo para subir e descer escadas 6 em crianças com PC. Entretanto, os ganhos obtidos com esses protocolos no componente de atividade foram inferiores aos ganhos obtidos na força muscular16 e alguns estudos randomizados, mais recentes, revelaram ganhos na força muscular (componente de estrutura e função do corpo) sem ganhos associados de mobilidade (componente de atividade).17-19

Exercícios específicos de fortalecimento muscular têm potencial para diminuir alterações nas estruturas e funções corporais que limitam o desempenho de atividades de mobilidade. $\mathrm{O}$ treinamento da força também pode ser realizado por meio de exercícios funcionais, segundo o princípio da especificidade. De acordo com esse princípio, o desempenho de tarefas motoras será otimizado por meio de um treinamento específico, diretamente relacionado à habilidade motora a ser adquirida, treinada num contexto relevante. ${ }^{20,21}$

Várias propostas de intervenção fisioterapêutica têm buscado incorporar condutas com objetivos funcionais mais claros, ou seja, objetivos que envolvem tarefas motoras do dia a dia, como por exemplo andar e subir escadas. 16,22 Algumas evidências sugerem maiores ganhos de funcionalidade após treinamento funcional comparado ao fortalecimento muscular isolado. 16,22 No entanto, ainda há escassez de informações empíricas em relação à documentação das mudanças individuais da função motora ao longo do tempo (i.e., perfil longitudinal de mudanças) em decorrência tanto do fortalecimento muscular isolado quanto do fortalecimento com tarefas funcionais. Detalhamento sobre o perfil de mudanças longitudinais decorrentes de protocolos de intervenção é necessário para pautar a definição dos parâmetros de administração dessas terapêuticas, tais 
como temporalidade e intensidade.

O estudo experimental de caso único é um desenho de pesquisa apropriado para a documentação sistemática de mudanças individuais ao longo do tempo, em resposta a uma intervenção, em contraste a desenhos experimentais tradicionais que avaliam efeito terapêutico em um grupo, ao final de uma intervenção. O objetivo deste estudo foi descrever mudanças na função motora de dois adolescentes com diplegia espástica, sendo cada adolescente participante de um protocolo de treinamento: fortalecimento muscular isolado (FMI) e fortalecimento com tarefas funcionais (FTF).

\section{Métodos}

Este estudo utilizou desenho experimental de caso único tipo $\mathrm{ABA} 23,24$ que permite a comparação dos dados da linha de base (A) com os resultados obtidos após a intervenção (B) e com os dados do follow-up pós-intervenção dos pacientes (A). Participaram do estudo duas adolescentes com PC, do sexo feminino. A participante I tinha quatorze anos de idade e a participante II doze anos tendo sua função motora grossa classificada nos níveis I e II, segundo o Sistema de Classificação da Função Motora Grossa (Gross Motor Function Classification SystemGMFCS), 25 respectivamente. Esta classificação estratifica crianças e adolescentes com PC de acordo com níveis de habilidades e limitações da função motora grossa. O GMFCS possui cinco níveis de classificação, que variam desde o nível I, que inclui as crianças mais hábeis, até o nível $\mathrm{V}$, que inclui as crianças com maior nível de comprometimento motor. Durante o período do estudo, os participantes não realizaram nenhuma outra terapia de reabilitação que buscasse o fortalecimento muscular.

Os critérios de inclusão no estudo foram: apresentar diagnóstico médico de diplegia espástica, ser capaz de deambular com ou sem dispositivo de auxílio e ser capaz de compreender comandos verbais simples. Os critérios de exclusão foram: não ter se submetido a outras intervenções tais como gesso seriado, toxina botulínica ou procedimentos cirúrgicos nos seis meses anteriores ao estudo, nem apresentar alterações cardíacas ou respiratórias que pudessem ser agravadas pelo exercício.

As fases de linha de base (i.e., baseline), intervenção e de pós-intervenção (i.e., follow-up) das participantes tiveram duração de quatro, seis e três semanas, respectivamente, totalizando uma período de acompanhamento longitudinal de 13 semanas. Medidas antropométricas (i.e., comprimento de pé, perna e coxa) foram realizadas antes e depois do estudo, segundo critérios de Li et al.,26 com o intuito de controlar possíveis influências do crescimento corporal nos resultados dos testes de força muscular.

O tipo de intervenção adminstrada foi definida por sorteio. A participante I foi submetida ao protocolo de fortalecimento muscular isolado (FMI), enquanto que a participante II foi submetida ao protocolo de fortalecimento muscular associado ao treinamento de tarefas funcionais (FTF). Os protocolos de intervenção foram aplicados durante período correspondente à fase $\mathrm{B}$ do estudo, três vezes por semana, com pelo menos um dia de intervalo entre as sessões, durante seis semanas. Ambos os protocolos foram dirigidos ao fortalecimento dos extensores do quadril, joelho e flexores plantares. Em ambos os protocolos, a sobrecarga foi aplicada por meio de pesos (caneleiras) com fecho ajustável de velcro.

O Protocolo do FMI, modificado a partir do protocolo de Dodd e Graham, 6 consistiu dos seguintes exercícios: a) com pesos ao redor da cintura e com os pés apoiados na borda de um degrau, realizar elevação na ponta dos pés; b) agachar e levantar com as costas apoiadas em uma bola, com pesos ao redor da cintura; c) com os pés um à frente do outro, agachar deslocando o corpo à frente, com peso ao redor da cintura (exercício feito bilateralmente); d) de pé, realizar flexão de quadril de forma a tirar o pé do chão e tocar o topo de um degrau, com peso ao redor do tornozelo (exercício feito bilateralmente); e) de pé sobre um degrau, realizar extensão de quadril tirando a perna do degrau e levando a perna para trás, com peso ao redor do tornozelo (exercício feito bilateralmente); e f) deitado em supino, com os joelhos e quadris flexionados, e pés apoiados na superfície, com peso sobre o abdomem, elevar a pelve da superfície e retornar.

O Protocolo de FTF, executado de acordo com o proposto por Blundell et al.,16 incluiu a execução dos seguintes movimentos: a) sentar e levantar de bancos de três alturas diferentes $(43,36$ e $30 \mathrm{~cm})$, com peso ao redor da cintura; b) agachar para pegar pequenos sacos de areia de aproximadamente 500 gramas, com peso ao redor da cintura; c) saltar em cama elástica com os dois pés juntos, apoiada pela mão, com peso ao redor da cintura; d) andar na ponta dos pés, com peso ao redor da cintura; e) subir e descer escada, com peso ao redor da cintura; f) caminhar na esteira, com peso ao redor da cintura.

A carga inicial foi estabelecida por meio da observação do desempenho do participante durante a execução da atividade e a progressão da mesma foi feita semanalmente, de forma a não permitir 
compensações na realização dos movimentos e fadiga muscular. Foram utilizados jogos e brincadeiras com o objetivo de estimular as adolescentes a obterem o melhor desempenho nos exercícios e atividades propostas.

As avaliações das adolescentes foram realizada três vezes por semana no laboratório de desempenho infantil da Fisioterapia e Terapia Ocupacional da Universidade Federal de Minas Gerais, em Belo Horizonte, entre janeiro e julho de 2007. Os profissionais responsáveis pelas avaliações (uma doutoranda e dois graduandos em fisioterapia) foram treinados para a utilização dos instrumentos e padronização de todas as medidas. Foram estabelecidos os índices de confiabilidade intra e inter-examinadores, tendo-se obtido índices de consistência 0,88 e 0,96 (i.e., coeficiente de Correlação Intraclasse 27 e Índice de Kappa,28 respectivamente), indicando a estabilidade das mensurações. Um único avaliador foi responsável pela obtenção das medidas de pico de força isométrica.

O componente de estrutura e função do corpo, conforme modelo da CIF, 8 foi avaliado por medidas de força de extensores do quadril, joelhos e flexores plantares. O componente de atividade foi analisado pelas medidas de velocidade da marcha, tempos para a criança se levantar do chão, subir e descer escadas e realizar o teste "Timed Get Up and Go" (TGUG).

$\mathrm{O}$ pico de força isométrica (Newtons) dos músculos extensores dos quadris e joelhos e dos flexores plantares foi mensurado com um dinamômetro manual5,6,9 da marca Microfet por um mesmo avaliador. Este valor foi convertido em torque (i.e., força multiplicada pelo comprimento do segmento, em Newton-metro), conforme dados antropométricos da participante. 26

Os procedimentos do teste de força máxima dos extensores de quadril, extensores do joelho e flexores plantares seguiram o protocolo determinado por Damiano e Abel, 5 como descrito a seguir. Cada adolescente foi solicitada a realizar uma primeira contração submáxima, para familiarização do procedimento, seguida de três contrações máximas, intervaladas com um minuto de repouso. Para as contrações máximas, as participantes foram instruídas a estender a articulação específica de forma a empurrar a mão do examinador (i.e., que posicionava o dinamômetro) com toda a força possível. Para cada grupo muscular, foi calculado o valor médio da força produzida nas três contrações máximas. A participante II não pôde realizar o teste de força dos flexores plantares pois não era capaz de produzir contrações suficientes desse grupo muscular para mover o tornozelo.

Para a aferição da velocidade de marcha (metros por segundo), solicitou-se às participantes percorrerem uma distância de dez metros em ritmo confortável utilizando os dispositivos usuais de auxílio para marcha. Os dois metros iniciais e finais do percurso foram desconsiderados por compreenderem períodos de aceleração e desaceleração do movimento. O tempo gasto para percorrer os seis metros intermediários foi registrado com o auxílio de um cronômetro. A média de duas tentativas foi calculada para esta e para todas as outras variáveis do componente de atividade.

Foi cronometrado o tempo (segundos) gasto para realização da tarefa de subida e descida de dez degraus de uma escada padrão. 29 As adolescentes foram solicitadas a realizar a tarefa o mais rápido possível, utilizando os corrimãos, se necessário. Foi permitido que as participantes escolhessem qualquer estratégia para a realização da tarefa, exceto subir/descer de lado.

Para a determinação do tempo para levantar do chão, que é um parâmetro sensível na determinação de alterações na mobilidade de crianças com desordens neurológicas, 30 as participantes, em decúbito dorsal e descalças, foram orientadas a se levantarem o mais rápido possível. A ação foi considerada concluída quando a adolescente, de pé, tocava um alvo colocado na altura da palma de sua mão e na linha média do corpo, com qualquer uma das mãos, braço totalmente elevado e cotovelo estendido.

A mobilidade foi igualmente avaliada pela aplicação do teste TGUG, um teste padrão utilizado para avaliar o nível geral de mobilidade em adultos e idosos. O TGUG consiste na medida de tempo (em segundos) gasto pelo indivíduo para completar a tarefa de levantar de uma cadeira, andar três metros, tocar um alvo, retornar e sentar novamente. Recentemente, um estudo demonstrou que esse teste possui alta confiabilidade também em crianças com incapacidade física. ${ }^{31} \mathrm{O}$ tempo para completar a tarefa foi calculado a partir do momento que a adolescente levantava-se do assento, apoiando com as mãos quando necessário. As participantes foram orientadas a caminharem o mais rápido possível, sem correr, até um suporte de madeira colocado a três metros do assento e encostarem a mão em um alvo fixado nesse suporte. Logo após, deveriam voltar e sentar-se novamente no assento, quando então era finalizada a contagem do tempo.

Em um estudo experimental de caso único, a análise das medidas intra e interfases torna-se necessária para a deteç̧ão de mudanças de 
comportamento na fase de intervenção, e se estas estão associadas ao tratamento proposto. ${ }^{24} \mathrm{As}$ mudanças observadas entre o primeiro e o último valor de todas as medidas das duas participantes foram descritas em termos percentuais.

O teste Shapiro-Wilk ${ }^{32}$ foi utilizado para testar a normalidade das distribuições das variáveis mensuradas, com um nível de significância de 0,05. A variabilidade dos dados, presença de tendência entre as fases e existência de dependência serial foram verificadas, respectivamente, pelo coeficiente de variação, estatística $\mathrm{C}$ (com ponto de corte de $1,64)^{23,24}$ e cálculo de auto-correlação. Os métodos estatísticos utilizados para analisar os dados referentes aos desfechos investigados foram Celeration Line e Banda de Dois DesviosPadrão.23,24

O método Celeration Line é utilizado em situações nas quais as medidas apresentam tendência de modificação, ou seja, os valores tendem a aumentar ou diminuir ao longo do tempo. Este método compara a tendência de modificação de uma determinada medida entre fases consecutivas do estudo. Para isso, é determinada uma linha que representa a tendência das medidas em uma fase do estudo. Em seguida, é feita a projeção dessa linha de tendência para a fase subsequente, e então é feita a contagem do número de medidas cujos valores estão acima ou abaixo da linha projetada. A ausência de mudanças de tendência entre fases subsequentes, é demonstrada pela proporção de dados acima e abaixo da linha permanece semelhante àquela observada na fase anterior. A significância estatística das mudanças de proporções é determinada através de tabela de probabilidades de do teste binomial, considerando o nível de significância $\alpha=0.05 .33$ Todas as medidas deste estudo foram analisadas pelo método Celeration Line.

A Banda de Dois Desvios Padrão é utilizada em situações em que as medidas de uma fase tem distribuição normal e não apresentam tendência de modificação. Esse método consiste em determinar, para uma fase do estudo, valores correspondentes a dois desvios-padrão acima e abaixo da média dos valores observados. Se, na fase seguinte, no mínimo duas medidas consecutivas estiverem ambas acima ou ambas abaixo dos limites superior e inferior da banda de dois desvios-padrão, mudanças entre as fases adjacentes serão consideradas significativas. Como a probabilidade de encontrar valores assim distribuídos é menor que 5\%, tal observação indica mudanças significativas entre fases com o nível de significância $\alpha=0,05.23,24$ este estudo, todas as medidas com distribuição normal e sem tendência também foram analisadas pelo método da Banda de Dois Desvios Padrão.

Por último, foi utilizada a análise visual das mudanças de cada medida ao longo das fases do estudo, conforme procedimentos padronizados descritos na literatura para caracterização dos níveis e da direção das mudanças, bem como a inclinação da linha de tendência das medidas. ${ }^{23}$ Para todas as variáveis (i.e., medidas de força muscular, velocidade de marcha, tempo para levantar, tempo para subir e descer escadas e TGUG), ganhos de desempenho durante a fase de intervenção ou perdas de desempenho durante a fase de pós-intervenção foram interpretadas como efeitos positivos do protocolo de treinamento utilizado. A análise visual foi feita por um examinador sem informações sobre os participantes e foi empregada como critério de desempate quando os dois métodos anteriores discordavam quanto à significância dos resultados. Os resultados descritos a seguir são aqueles referentes ao método (Celeration Line ou Banda de Dois Desvios Padrão) condizente com a análise visual.

Os procedimentos do presente estudo foram aprovados pelo Comitê de Ética em Pesquisa da Universidade Federal de Minas Gerais (CEP-UFMG, parecer 219/07) e o Termo de Consentimento Livre e Esclarecido foi assinado pelos responsáveis anteriormente à inclusão no estudo.

\section{Resultados}

Ambas as participantes compareceram em todas as sessões de avaliação e de intervenção. As medidas antropométricas do comprimento dos pés, perna e coxas das participantes não apresentaram modificações estatisticamente significativas durante o estudo, indicando ausência de influência desses fatores nos torques musculares gerados ao longo do estudo.

Os resultados apresentados a seguir são reportados para cada participante, separadamente. As Tabelas 1 e 2 contém os resultados dos métodos de análise Celeration Line ou Banda de Dois Desvios Padrão das participantes I e II (no caso de conflito nos resultados fornecidos pelos dois métodos, prevaleceu o que foi consistente com a Análise Visual). No desenho experimental de caso único, o comportamento das medidas ao longo do tempo é o que permite compreender o perfil de mudanças que caracteriza os efeitos de uma intervenção. Diferenças percentuais entre o primeiro e último valor das medidas realizadas não podem ser analisadas de forma isolada, ou seja, só podem ser ana- 
Figura 1

Comportamento longitudinal para a participante I nas variáveis relacionadas às estruturas e funções do corpo (força muscular) e atividade (tarefas funcionais) nas três fases do estudo. Belo Horizonte, 2007.
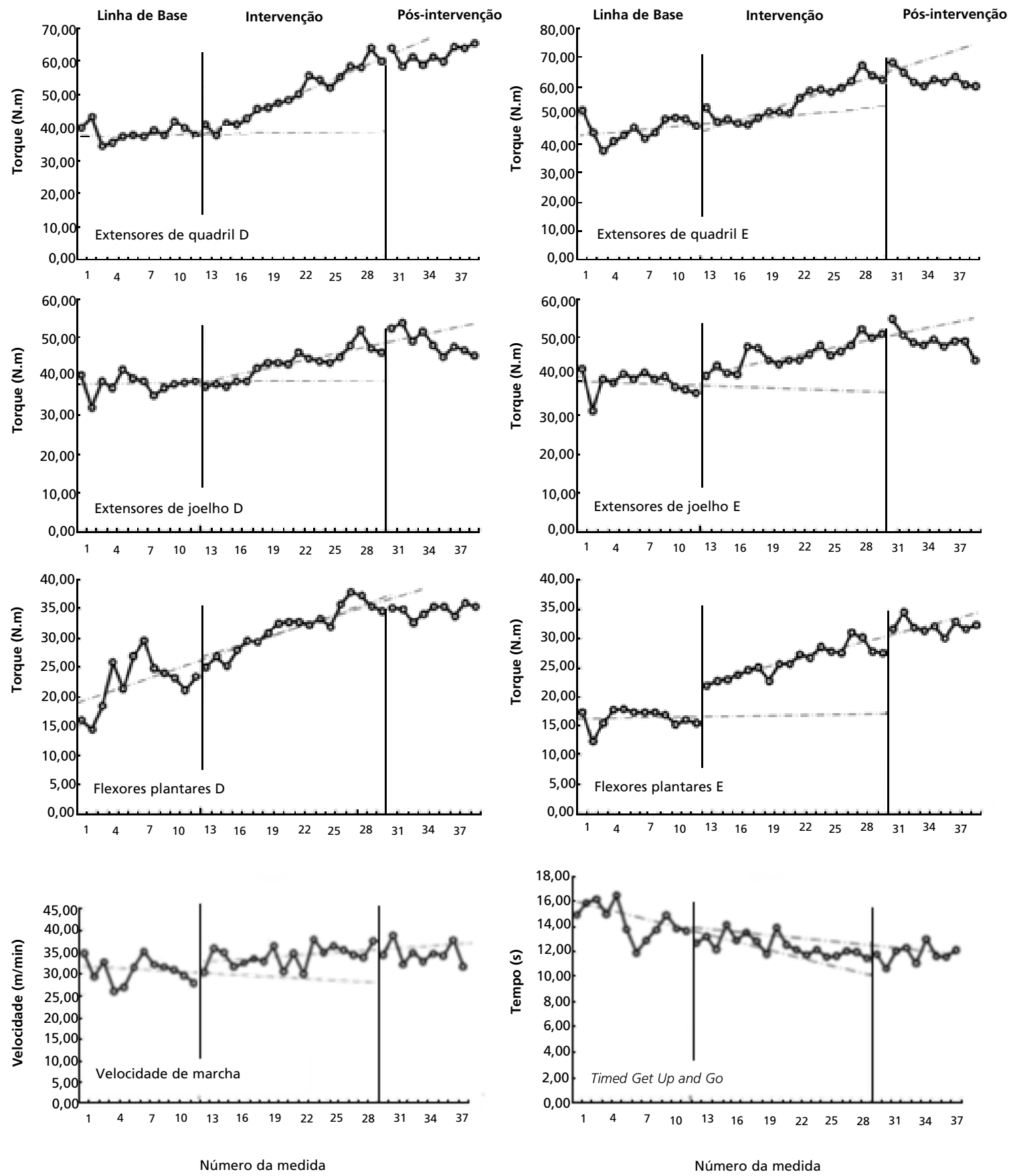

continua

Linha tracejada: Celeration Line, que se prolonga para a fase subsequente; $D=$ referente ao membro inferior direito; $\quad E=$ referente ao membro inferior esquerdo. 
Comportamento longitudinal para a participante I nas variáveis relacionadas às estruturas e funções do corpo (força muscular) e atividade (tarefas funcionais) nas três fases do estudo. Belo Horizonte, 2007.

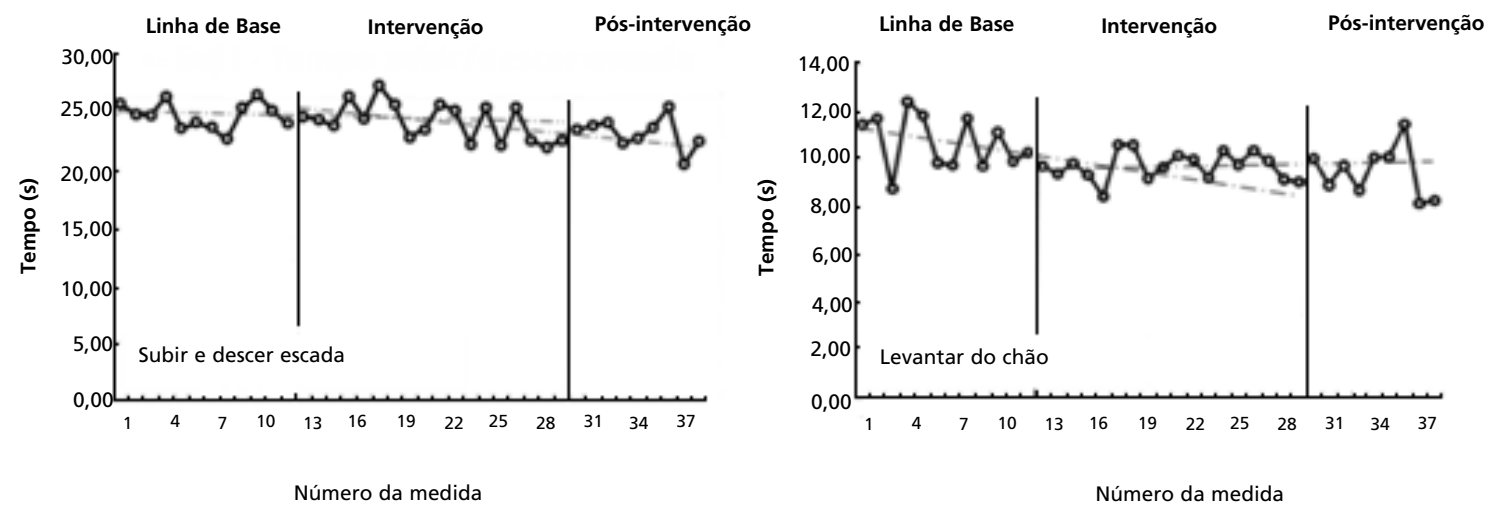

Linha tracejada: Celeration Line, que se prolonga para a fase subsequente; $D=$ referente ao membro inferior direito; $\mathrm{E}=$ referente ao membro inferior esquerdo.

\section{Figura 2}

Comportamento longitudinal para a participante II nas variáveis relacionadas às estruturas e funções do corpo (força muscular) e atividade (tarefas funcionais) nas três fases do estudo. Belo Horizonte, 2007.
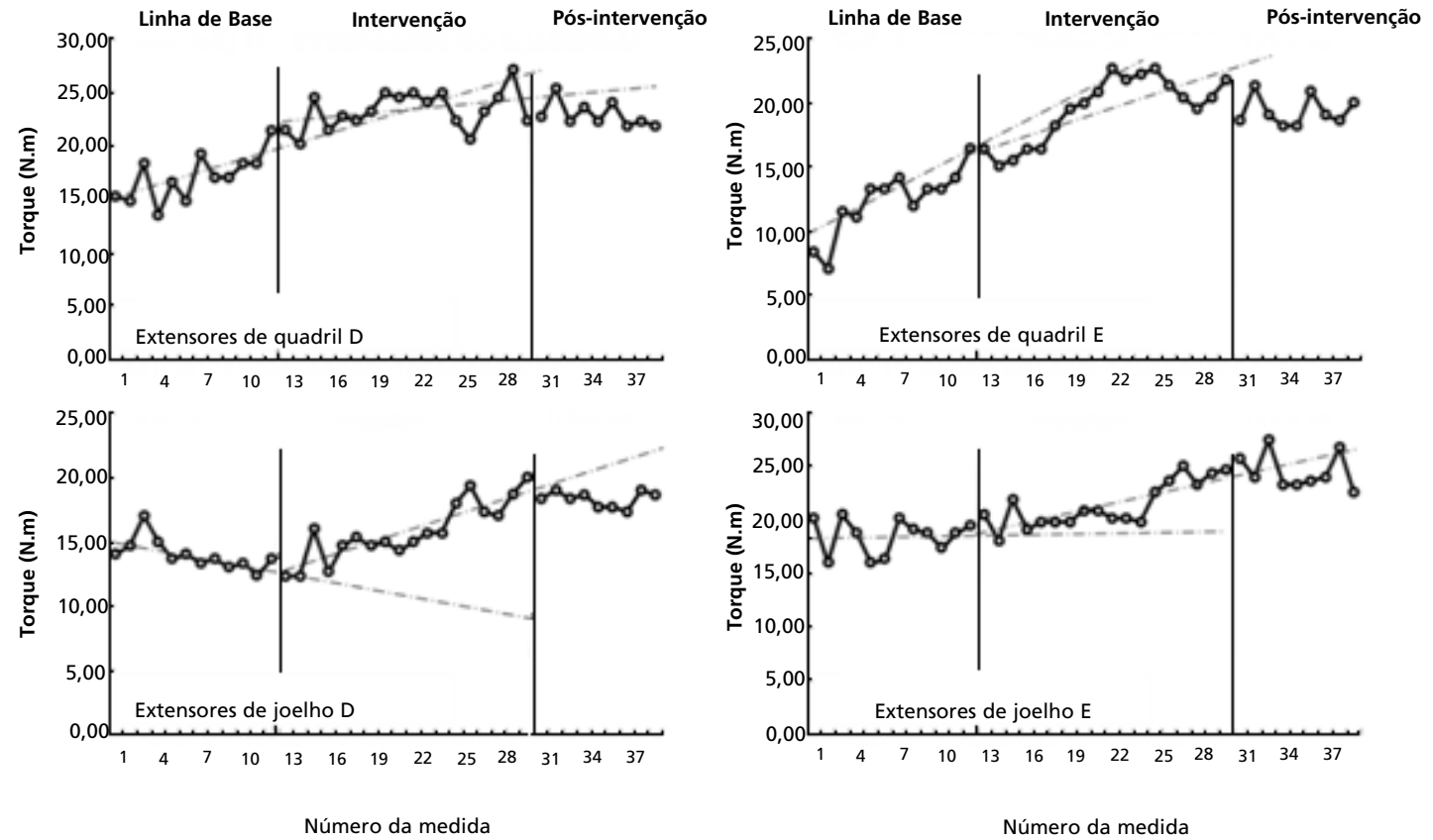

continua

Linha tracejada: linha de aceleração (Celeration Line), que se prolonga para a fase subsequente; $D=$ referente ao membro inferior direito; $\mathrm{E}=$ referente ao membro inferior esquerdo. 
Comportamento longitudinal para a participante II nas variáveis relacionadas às estruturas e funções do corpo (força muscular) e atividade (tarefas funcionais) nas três fases do estudo. Belo Horizonte, 2007.
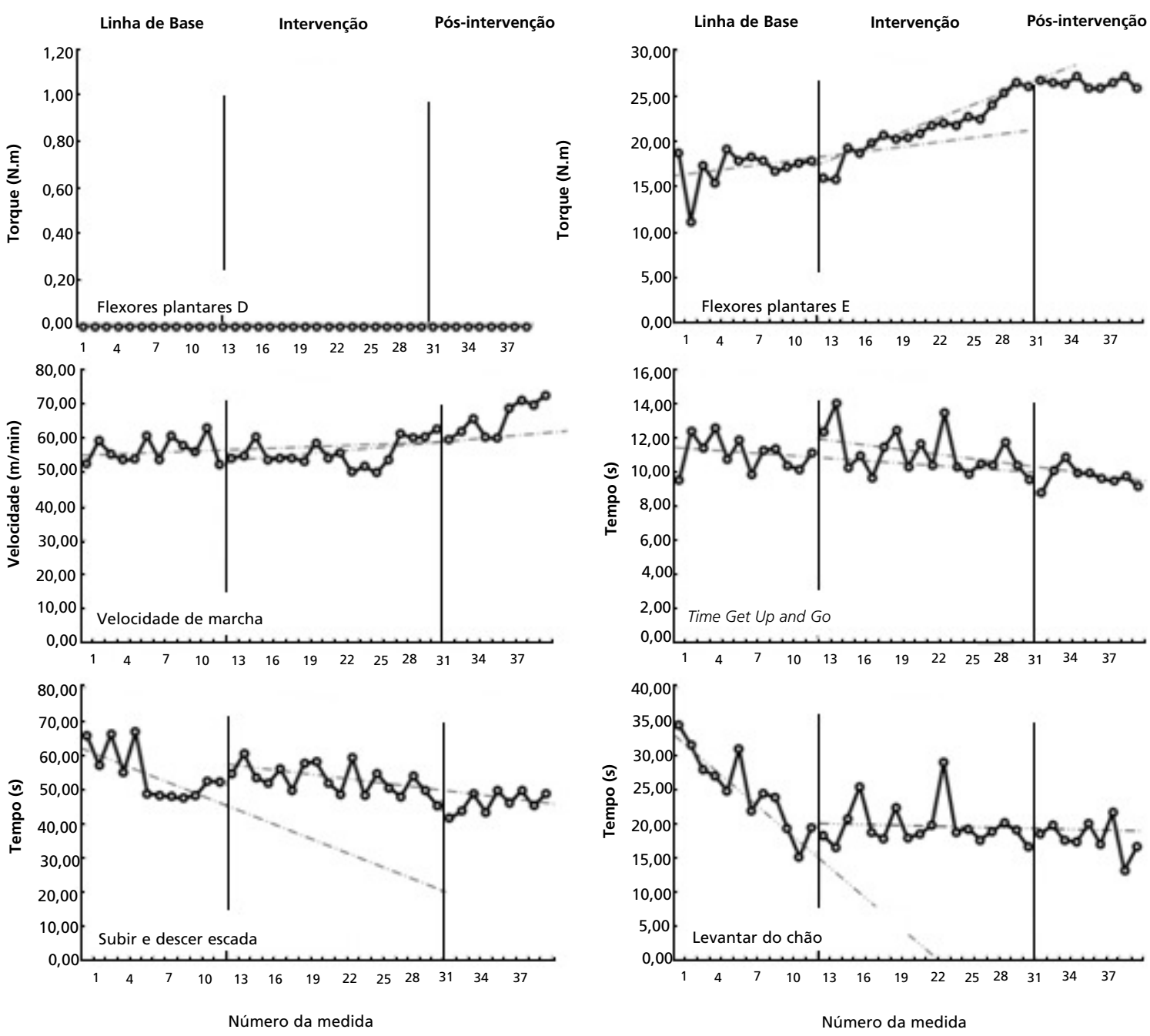

Linha tracejada: linha de aceleração (Celeration Line), que se prolonga para a fase subsequente; $D=$ referente ao membro inferior direito; $E=$ referente ao membro inferior esquerdo.

lisadas no contexto das mudanças longitudinais da medida em questão. Por isso, apenas percentuais de medidas que apresentaram mudanças significativas ao longo das fases do estudo são reportadas a seguir. Os valores percentuais de mudança entre a primeira e última medida de todas as variáveis estão reportadas na Tabela 3.

A participante I, submetida ao FMI, apresentou aumento de força estatisticamente significativo em todos os grupos musculares avaliados, exceto nos extensores de quadril esquerdo quando a intervenção foi comparada à linha de base (Tabela 1). Conforme demonstrado pela tendência de mudança das medidas, ao longo do período pós-intervenção, a tendência de aumento da força muscular observada durante a intervenção em geral não se manteve (Figura 1). A mudança de tendência foi significativa para os extensores de quadril direito e esquerdo e para os flexores plantares direito (Tabela 1). Todos esses resultados suportam a interpretação de que a intervenção promoveu ganhos reais na força muscular. As diferenças percentuais entre a última e a primeira coleta mostraram ganhos de $63 \%$ para os extensores de quadril direito, $13 \%$ e $5 \%$ para exten- 
Tabela 1

Efeito da aplicação do protocolo fortalecimento muscular isolado sobre os componentes de funcionalidade (estrutura e função do corpo e atividade) na participante I.

\begin{tabular}{|c|c|c|c|c|}
\hline \multirow{2}{*}{$\begin{array}{l}\text { Componente de funcionalidade } \\
\text { Estrutura e função do corpo (for } \\
\text { muscular) }\end{array}$} & \multicolumn{2}{|c|}{$\begin{array}{l}\text { Intervenção comparada à } \\
\text { Linha de Base }\end{array}$} & \multicolumn{2}{|c|}{$\begin{array}{c}\text { Pós-intervenção comparada } \\
\text { à Intervenção }\end{array}$} \\
\hline & & & & \\
\hline Extensores quadril D & * & $\uparrow>$ & * & $\uparrow<$ \\
\hline Extensores quadril E & NS & $\uparrow>$ & * & $\downarrow<$ \\
\hline Extensores joelho $D$ & * & $\uparrow>$ & NS & $\downarrow<$ \\
\hline Extensores joelho E & * & $\uparrow>$ & NS & $\downarrow<$ \\
\hline Flexores plantares $\mathrm{D}$ & * & $\uparrow>$ & * & $\rightarrow<$ \\
\hline Flexores plantares E & * & $\uparrow>$ & NS & $\rightarrow<$ \\
\hline \multicolumn{5}{|l|}{ Atividade } \\
\hline Levantar do chão & NS & - & NS & $\rightarrow>$ \\
\hline TGUG & * & $\uparrow<$ & * & $\rightarrow<$ \\
\hline Velocidade de marcha & NS & - & NS & - \\
\hline Subir e descer escada & NS & $\uparrow<$ & NS & - \\
\hline
\end{tabular}

Tabela 2

Efeito da aplicação do protocolo fortalecimento com tarefas funcionais sobre os componentes de funcionalidade (estrutura e função do corpo e atividade) na participante II.

\begin{tabular}{|c|c|c|c|c|}
\hline \multirow{3}{*}{$\begin{array}{l}\text { Componente de funcionalidade } \\
\text { Estrutura e função corpo (força } \\
\text { muscular) }\end{array}$} & \multicolumn{2}{|c|}{$\begin{array}{l}\text { Intervenção comparada à } \\
\text { Linha de Base }\end{array}$} & \multicolumn{2}{|c|}{$\begin{array}{c}\text { Pós-intervenção comparada } \\
\text { à Intervenção }\end{array}$} \\
\hline & & & & \\
\hline & & & & \\
\hline Extensores quadril D & * & $\uparrow<$ & * & $\downarrow<$ \\
\hline Extensoresquadril E & NS & $\uparrow>$ & * & $\uparrow<$ \\
\hline Extensores joelho D & * & $\uparrow>$ & * & $\rightarrow<$ \\
\hline Extensores joelho E & NS & $\uparrow<$ & NS & $\downarrow<$ \\
\hline Flexores plantares $D$ & - & & - & \\
\hline Flexores plantares E & * & $\uparrow>$ & NS & $\rightarrow<$ \\
\hline \multicolumn{5}{|l|}{ Atividade } \\
\hline Levantar do chão & * & $\downarrow<$ & NS & $\uparrow>$ \\
\hline TGUG & NS & - & NS & - \\
\hline Velocidade de marcha & NS & - & * & $\uparrow>$ \\
\hline Subir e descer escada & * & $\uparrow<$ & * & $\rightarrow<$ \\
\hline
\end{tabular}


Estatística descritiva das mudanças entre a primeira e última medidas (em porcentagem) para as variáveis relacionadas a estruturas e funções do corpo (força muscular) e atividade (tarefas funcionais) para ambas as participantes. Belo Horizonte, 2007.

\begin{tabular}{lcc}
\hline Componente de funcionalidade & \multicolumn{2}{c}{$\begin{array}{c}\text { Diferença percentual entre a primeira e a última } \\
\text { medidas }\end{array}$} \\
\cline { 2 - 3 } & Participante I & Participante II \\
\cline { 2 - 3 } & & \\
\hline Estrutura e função do corpo (força & & $\uparrow 43,0$ \\
muscular) & $\uparrow 63,0$ & $\uparrow 137,0$ \\
Extensores quadril D & $\uparrow 16,0$ & $\uparrow 33,0$ \\
Extensores quadril E & $\uparrow 13,0$ & $\uparrow 12,0$ \\
Extensores joelho D & $\uparrow 5,0$ & $\uparrow 0,0$ \\
Extensores joelho E & $\uparrow 122,0$ & $\uparrow 38,0$ \\
Flexores plantares D & $\uparrow 86,0$ & \\
Flexores plantares E & & $\downarrow 52,0$ \\
& & $\downarrow$ \\
Atividade & $\downarrow 28,0$ & $\uparrow, 0$ \\
Levantar do chão & $\downarrow 19,0$ & $\downarrow 38,0$ \\
TGUG & $\downarrow 9,0$ & $\downarrow 26,0$ \\
Velocidade de marcha & $\downarrow 13,0$ & \\
Subir e descer escada & & \\
\hline
\end{tabular}

$\uparrow:$ indica ganho percentual. $\downarrow$ : indica perda percentual; $D=$ direito; $E=$ esquerdo; TGUG= Timed Get Up and Go.

sores do joelho direito e esquerdo respectivamente e $122 \%$ e $86 \%$ para flexores plantares direito e esquerdo, respectivamente (Tabela 2).

Para as medidas de atividade, os ganhos na velocidade de marcha, no tempo de subir e descer escadas, e no tempo de levantar-se do chão não alcançaram significância em nenhuma das comparações entre fases (Tabela 1). Para o TGUG, foi observada uma diminuição progressiva no tempo para realizar a atividade durante a intervenção. No entanto, essa tendência foi menos intensa do que a que já vinha sendo observada nas medidas da linha de base (Figura 1). Ao longo da fase pós-intervenção, o desempenho se estabilizou. A diferença entre o pós-intervenção e a intervenção para essa variável também foi significativa (Tabela1). A diferença percentual do tempo para completar o TGUG entre a primeira e a última avaliações mostrou uma redução $19 \%$.

A participante II, submetida ao FTF, apresentou aumento de força estatisticamente significativo dos extensores de quadril e joelho do lado direito e dos flexores plantares esquerdos (Tabela 2) ao longo da intervenção. Os outros grupos musculares não apresentaram ganhos significativos de força. Ao longo do pós-intervenção, a tendência de ganho de força que vinha sendo observada durante a intervenção em geral não se manteve (Figura 2). As diferenças dessa última comparação foram significativas para os extensores de quadril, bilateralmente, e os extensores de joelho direito (Tabela 2 e Figura 2). Os ganhos percentuais entre a última e a primeira coleta foram de $43 \%$ para os extensores de quadril direito, $137 \%$ para os extensores de quadril esquerdo, $33 \%$ para extensores do joelho direito e $38 \%$ para flexores plantares do lado esquerdo (Tabela 3).

Para as medidas de atividade, ao longo da intervenção foram observadas mudanças significativas em comparação com a linha de base nas medidas do tempo gasto para levantar do chão e subir e descer escadas (Tabela 2). As mudanças ocorreram no sentido contrário ao esperado, no entanto. Conforme ilustrado na Figura 2, durante a intervenção a tendência de melhora de desempenho (redução do tempo) nessas atividades foi menos intensa do que a que vinha sendo observada na linha de base. Ao longo do pós intervenção, o tempo gasto para subir e descer escadas tendeu a estabilizar, em comparação com a tendência de redução observada durante a intervenção (Figura 2). Essa diferença foi significativa (Tabela 2). Para a velocidade de marcha, o pós intervenção revelou ganhos de desempenho adicionais à tendência observada na intervenção (Figura 2), diferença que também foi significativa (Tabela 2). As diferenças percentuais entre a primeira e a última medidas do tempo gasto para 
levantar do chão e subir e descer escadas indicaram redução de $52 \%$ e $26 \%$ respectivamente. As mesmas medidas de velocidade de marcha mostraram um aumento de $38 \%$.

\section{Discussão}

Este estudo apresenta dados prospectivos sobre os efeitos terapêuticos de dois protocolos de fortalecimento muscular na reabilitação de duas adolescentes com diplegia espástica. Em geral, os ganhos observados para ambas as participantes foram mais consistentemente positivos no nível das estruturas e funções do corpo (nas medidas de força muscular) do que no nível de atividade (nas medidas de velocidade de marcha, tempo pra subir e descer escadas, levantar do chão, completar o TGUG e na velocidade de marcha). Esses achados sugerem que, apesar da relação já demonstrada entre força e função em crianças com PC,34 os ganhos de desempenho no nível de estrutura e função do corpo não se traduzem de maneira linear em ganhos de atividades. $O$ desenho experimental de caso único é ideal para registrar detalhadamente as mudanças de desempenho apresentadas por seus participantes ao longo do tempo. Apesar do tipo de desenho metodológico não permitir comparações diretas entre os protocolos de treinamento usados para cada participante, o perfil de mudanças ao longo do tempo sugere interpretações relevantes a cerca dos efeitos de cada protocolo de treinamento.

A participante I teve ganhos mais consistentes de força dos grupos musculares testados, o que condiz com o protocolo de FMI utilizado. Um artigo de revisão sistemática13 realizado em 2008 apontou que programas de treinamento de força, aplicados durante seis semanas, com frequência de três sessões semanais, são suficientes para melhorar o torque dos músculos dos membros inferiores. Os protocolos dos estudos revisados se assemelham ao FMI realizado neste estudo, o que corrobora os ganhos de força observados para a participante I. Diversos outros estudos fornecem evidências da efetividade do treinamento muscular específico para o ganho de força.4,5,6,7,9,10,11,16,22 Já os ganhos funcionais da participante I foram pouco expressivos. A análise visual do perfil de mudanças dessa participante sugere uma pequena tendência geral de ganho de desempenho nas atividades funcionais investigadas, mas essa tendência somente alcançou significância no tempo gasto para completar o TGUG.

A participante II, que realizou o FTF, teve ganhos menos consistentes na força muscular. Estudos de Blundell et al.16 e Liao et al.,22 no entanto, mostraram a efetividade de programas funcionais de fortalecimento (com treino na esteira ou treino de sentar e levantar com sobrecarga no tronco) na capacidade de gerar força nas musculaturas dos membros inferiores em crianças com diplegia espástica. Assim, é possível que a participante II tivesse tido ganhos mais expressivos de força com um protocolo mais agressivo em termos de definição do grau de sobrecarga e da sua modificação ao longo do tempo. Além disso, é importante ressaltar que a participante não apresentava movimentação ativa em flexão plantar do tornozelo direito, o que comprometeu a possibilidade de ganho de força desse grupo muscular. As modificações de desempenho nas atividades funcionais da participante II também mostram uma tendência geral de melhora ao longo do tempo (Figura 2). Os resultados da análise dos dados, no entanto, mostram que essa tendência de ganho de já vinha sendo apresentada durante a linha de base (antes do início da intervenção), e perdeu intensidade durante o treinamento.

A tendência de ganho funcional já na fase de linha de base dificulta a demonstração de efeitos positivos para qualquer tipo de intervenção. Isso ocorre porque tal tendência de ganho de desempenho nos testes utilizados ocorre naturalmente, devido à repetição que possibilita o aprendizado dos testes. Quando a intervenção começa, boa parte do ganho de desempenho já ocorreu devido ao aprendizado. Essa é uma limitação e é ao mesmo tempo uma vantagem dos métodos de acompanhamento longitudinal do desenho experimental de caso único. Por um lado, é preciso considerar que em ensaios clínicos controlados, participantes de grupos experimental e controle são testados usualmente uma única vez antes e uma segunda vez depois do tratamento. Como situações de teste normalmente envolvem aprendizado, ganhos entre as medidas pré e pós tratamento podem ser decorrentes do aprendizado, pelo menos parcialmente, ao invés da intervenção. Por outro lado, com medidas longitudinais, a tendência de mudança ao longo do tempo na linha de base fica determinada antes do início de qualquer intervenção. Isso dificulta detectar ganhos adicionais produzidos pela intervenção, ao mesmo tempo que permite interpretações mais cautelosas.

No presente estudo, a repetição das medidas dificultou a diferenciação dos efeitos específicos de cada protocolo na funcionalidade das adolescentes, uma vez que as duas adolescentes realizaram, sistemática e repetidamente, tanto medidas de força muscular quanto as atividades funcionais. Dessa forma, essas mensurações repetidas configuraram-se como uma espécie de treinamento tanto específico 
(de força muscular) quanto funcional (de atividades) em ambos os protocolos. Isso foi evidenciado pela tendência ganhos tanto no nível de estrutura e função quanto nas atividades antes do início da intervenção. É preciso também considerar a interpretação das mudanças observadas após o término das intervenções. Três possibilidades devem ser consideradas. Após o término da fase de intervenção, o desempenho em determinada medida pode apresentar, ao longo do tempo, um (a) padrão de queda, (b) manutenção ou (c) melhora. Em geral, a queda de desempenho após a suspensão de uma intervenção é interpretada como evidência de seus efeitos positivos, porém transitórios (que são demonstrados somente quando a intervenção está presente). A manutenção do desempenho após o fim da intervenção, em nível superior àquele observado antes de seu início, pode indicar a produção de efeitos duradouros. Uma tendência de melhora após o fim da intervenção, por último, pode sugerir que os ganhos de desempenhos não sejam decorrentes da intervenção em si, e sim de um processo mais longo de aprendizagem.

No presente estudo, conforme ilustrado nas Figuras 1 e 2 e Tabelas 1 e 2, as medidas de força que apresentaram diferenças significativas entre o período pós-intervenção e a intervenção desenvolveram os três padrões possíveis ( $\mathrm{a}, \mathrm{b}$ e c). Tanto o efeito do destreinamento, consistente com o padrão (a) quanto o efeito da aprendizagem dos testes, condizente com o padrão (c), são documentados na literatura para crianças com paralisia cerebral espástica. 13

O padrão (b), consistente com a interpretação de ganho duradouro de força muscular, também foi observado. A definição das características de treinamento, como sua frequência, duração, cargas aplicadas e período máximo de suspensão sem perdas parecem variar não só de indivíduo para indivíduo, mas também de grupo muscular para grupo muscular. Essa variação entre grupos musculares provavelmente se relaciona ao padrão de uso típico dos diferentes grupos musculares nas atividades do dia a dia. Na clínica de fisioterapia, medidas padronizadas periódicas da força muscular seriam muito úteis para garantir a escolha de parâmetros do treinamento esteja de fato promovendo ganhos contínuos ou pelo menos manutenção da força muscular.

Para as atividades funcionais que apresentaram diferenças significativas entre o período pós-intervenção e a intervenção, duas mostraram manutenção dos ganhos (b) após a intervenção (TGUG da parti- cipante I e tempo para subir e descer escadas da participante II). A velocidade de marcha da participante II apresentou o padrão de melhora (c) após o término da intervenção. Esta observação também abre uma perspectiva interessante para a prática clínica: a documentação dos efeitos de um programa de treinamento precisa se estender além do momento da alta do paciente, e incluir um período de seguimento. Isso precisa ser feito não pela possibilidade já apontada do destreinamento, mas também pela possibilidade da incorporação tardia de efeitos do treinamento nas tarefas de vida diária, como parece ter sido o caso da velocidade de marcha. É plausível que o indivíduo consiga ir transferindo, ao longo do tempo, ganhos das estruturas e funções corporais promovidos de maneira mais concreta pelo treinamento para ganhos nas suas atividades.

O presente estudo é limitado por características inerentes à metodologia experimental de caso único. A repetição de testes se sobrepõe ao programa de treinamento e pode confundir seus efeitos. A necessidade de testes em ambos os protocolos também reduz as diferenças entre eles e dificulta determinar efeitos específicos de cada um. A caracterização do padrão de mudanças ao longo do tempo, no entanto, permite uma riqueza de análise normalmente não vista em estudos feitos com grupos de pacientes, identificando padrões de aprendizagem, destreinamento e ganhos funcionais tardios. A metodologia é simples, baseada em aplicação sistemática de avaliações periódicas e, se inserida na rotina clínica, poderia enriquecer o entendimento do clínico sobre o progresso de seus pacientes.

Os resultados do presente estudo demonstraram que a associação de testes repetidos com o treino de fortalecimento muscular (específico e funcional) promoveram benefícios clínicos em crianças e adolescentes com PC. Apesar dos ganhos observados, não é possível afirmar que esses possam ser atribuídos exclusivamente aos protocolos de fortalecimento muscular utilizados, especialmente porque as crianças começaram a demonstrar ganhos antes do início das intervenções, refletindo um aprendizado em decorrência das mensurações repetidas. De qualquer modo, a utilização clínica de testes sistemáticos repetidos pode ser útil, tanto porque a associação entre protocolos de fortalecimento e testes funcionais repetidos poderia promover aquisição de habilidades motoras em crianças com PC, quanto porque os testes repetidos permitiriam ao clínico compreender o padrão de mudança de seus pacientes em resposta às suas propostas de tratamento. 


\section{Referências}

1. Bax M, Goldstein M, Rosenbaum P, Leviton A, Paneth N, Dan B, Jacobsson B, Damiano D; Executive Committee for the Definition of Cerebral Palsy. Proposed definition and classification of cerebral palsy. Dev Med Child Neurol. 2005; 47 (8): 571-6.

2. Howle JMW. Cerebral Palsy. In: Campbell SK, editor. Decision making in pediatric neurologic physical therapy. Philadelphia: Churchhill Livingstone; 1999. p. 23-83.

3. Sankar C, Mundkur, N. Cerebral palsy: definition, classification, etiology and early diagnosis. Indian J Pediatr. 2005; 72 (10): 865-8.

4. Eagleton M, Iams A, McDowell J, Morrison R, Evans CL.The effects of strength training on gait in adolescents with cerebral palsy. Ped Phys Ther. 2004; 16 (1): 22-30.

5. Damiano DL, Abel MF. Functional outcomes of strength training in spastic cerebral palsy. Arch Phys Med Rehabil. 1998; 79 (2): 119-25.

6. Dodd KJ, Graham HK. A randomized clinical trial of strength training in young people with cerebral palsy. Develop Med Child Neurol. 2003; 45(10): 652-7.

7. Mulligan $\mathrm{H}$. The outcome of a functional exercise programme in an adolescent ith cerebral palsy: a single case study. NZ J Physiother. 2004; 32 (1): 30-8.

8. OMS (Organização Mundial da Saúde). CIF: Classificação Internacional de Funcionalidade, Incapacidade e Saúde. São Paulo: EDUSP; 2003.

9. Jung JW, Her JG, Ko J. Effect of strength training of ankle plantarflexors on selective voluntary motor control, gait parameters, and gross motor function of children with cerebral palsy. Phys Ther Sci. 2013; 25 (10): 1259-63.

10. Damiano DL, Kelly LE, Vaughn CL. Effects of quadriceps femoris muscle strengthening on crouch gait in children with spastic diplegia. Phys Ther. 1995; 75 (8): 658-67.

11. Morton JF, Brownlee M, Mc Fadyen A. The effects of progressive resistance training for children with cerebral palsy. Clin Rehabil. 2005; 19 (3): 283-9.

12. Bobath $\mathrm{K}$. The normal postural reflex mechanism and its deviation in children with cerebral palsy. Physiotherapy. 1971; 57 (11): 515-25.

13. Verschuren O, Ketelaar M, Takkenz T, Helders PJ, Gorter JW. Exercise programs for children with cerebral palsy: a systematic review of the literature. Am J Phys Med Rehabil. 2008; 87 (5): 404-17.

14. Fowler EG, Ho TW, Nwigwe AI, Dorey FJ. The effect os quadriceps femoris muscle strenhtening exercises on spasticity in children with cerebral palsy. Phys Ther. 2001; 81 (6): 1215-23.

15. Andersson C, Grooten W, Hellsten M, Kaping K, Mattsson E. Adults with cerebral palsy: walking ability after progressive strength training. Develop Med Child Neurol. 2003; 45 (4): $220-8$.

16. Blundell SW, Shepherd RB, Dean CM, Adams RD, Cahill BM. Functional strength training in cerebral palsy: a pilot study of a group circuit training class for children aged 4-8 years. Clin Rehabil. 2003; 17 (1): 48-57.

17. Taylor NF, Dodd KJ, Baker RJ, Willoughby K, Thomason
P, Graham HK. Progressive resistance training and mobility-related function in young people with cerebral palsy: a randomized controlled trial. Dev Med Child Neurol. 2013; 55 (9): 806-12.

18. Scholtes VA, Becher JG, Comuth A, Dekkers H, Van Dijk L, Dallmeijer AJ. Effectiveness of functional progressive resistance exercise strength training on muscle strength and mobility in children with cerebral palsy: a randomized controlled trial. Dev Med Child Neurol. 2010; 52 (6): 10713.

19. Boyd RN. Functional progressive resistance training improves muscle strength but not walking ability in children with cerebral palsy. J Physiother. 2012; 58 (3): 197.

20. Salem Y, Godwin EM. Effects of task-oriented training on mobility function in children with cerebral palsy. NeuroRehabilitation. 2009; 24 (4): 307-13.

21. Scholtes VA, Becher JG, Janssen-Potten YJ, Dekkers H, Smallenbroek L, Dallmeijer AJ. Effectiveness of functional progressive resistance exercise training on walking ability in children with cerebral palsy: a randomized controlled trial. Res Dev Disabil. 2012; 33 (1): 181-8.

22. Liao HF, Liu YC, Liu WY, Lin YT. Effectiveness of loaded sit-to-stand resistance exercise for children with mild spastic diplegia: a randomized clinical trial. Arch Phys Med Rehabil. 2007; 88 (1): 25-31.

23. Nourbakhsh MR, Ottenbacher KJ. The statistical analysis of single-subject data: a camparitive examnination. Phys Ther. 1994; 74 (8): 768-76.

24. Richards SB, Taylor R, Ramasamy R, Richards RY. Singlesubject research: application in educational and clinical settings. San Diego: Singular Pub. Group; 1999.

25. Hiratuka E, Matsukura TS, Pfeifer LI. Cross-cultural adaptation of the Gross Motor Function Classification System into Brazilian-Portuguese (GMFCS). Rev Bras Fisioter. 2010; 14 (6): 537-44.

26. Li RC, Jasiewicz JM, Middleton J, Condie P, Barriskill A, Hebnes H, Purcell B. The development, validity, and reliability of a manual muscle testing device with integrated limb position sensors. Arch Phys Med Rehabil. 2006; 87 (3): 411-7.

27. Bravo G, Potvin L. Estimating the reliability of continuous measures with Cronbach's alpha or the Intraclass correlation coefficient: Toward the integration of two traditions. J Clin Epidemiol. 1991; 44(4/5): 381-90

28. Sim J, Wright CC. The Kappa statistic in reliability studies: Use, interpretation, and sample size requirements. Phys Ther. 2005; 85(3): 257-68.

29. Zaino CA, Marchese VG, Westcott SL. Timed up and down stairs test: preliminary reliability and validity of a new measure of functional mobility. Pediatr Phys Ther. 2004; 16 (2): $90-8$.

30. Beenakker EA. Functional ability and muscle force in healthy children and ambulant Duchenne muscular dystrophy patients. Eur J Paediatr Neurol. 2005; 9: 387-93.

31. Williams EN, Carroll SG, Reddihough DS, Phillips BA, Galea MP. Investigation of the timed 'Up \& Go' test in children. Dev Med Child Neurol. 2005; 47 (8): 518-24. 
32. Razali NM, Wah YB. Power comparisons of Shapiro-Wilk, Kolmogorov-Smirnov, Lilliefors and Anderson-Darling tests. J Statistical Modeling Analytics. 2011; 2 (1): 21-33.

33. Nourbakhsh MR, Ottenbacher KJ. The statistical analysis of single-subject data: a comparative examination. Physical Therapy. 1994; 74(8): 768-76

Recebido em 9 de junho de 2014

Versão final apresentada em 29 de outubro de 2014

Aprovado em 30 de dezembro de 2014
34. Ross SA, Engsberg JR. Relationships between spasticity, strength, gait, and the GMFM-66 in persons with spastic diplegia cerebral palsy. Arch Phys Med Rehabil. 2007; 88 (9): 1114-20 